\title{
Avaliação isocinética do joelho no pré-operatório de artroplastia total
}

\section{Isokinetic evaluation of the knee in the preoperative of total arthroplasty}

\author{
Carolina Barreto Mozzini ${ }^{1}$; Daiana Moreira Mortari ${ }^{2}$; Gilnei Lopes Pimentel ${ }^{3}$ \\ ${ }^{1}$ Fisioterapeuta - UPF, Mestre em Ciências - Oncologia - USP, Doutoranda em Ciências - Oncologia - USP. \\ ${ }^{2}$ Fisioterapeuta - UPF. \\ ${ }^{3}$ Professor Mestre do curso de Fisioterapia - UPF. \\ Endereço para correspondência \\ Carolina Barreto Mozzini \\ R. Teixeira Soares, 1392 - Centro \\ 99010-081 - Passo Fundo - RS [Brasil] \\ carolmozzini@gmail.com
}

\begin{abstract}
Resumo
A osteoartrose de joelho dificulta as atividades rotineiras e a deambulação pela degeneração articular e alterações do desempenho muscular que o membro inferior afetado apresenta. Neste artigo, objetivou-se analisar retrospectivamente o pico de torque muscular isocinético concêntrico dos extensores e flexores do joelho, por meio da dinamometria computadorizada. Compararam-se 13 indivíduos com osteoartrose de joelho, ocasionada pela degeneração da cartilagem, no pré-operatório de artroplastia total unilateral, com outros 13 sem essa doença. Os achados mostram que os portadores dessa enfermidade apresentam redução significativa do pico de torque de flexores e extensores do joelho no membro acometido, quando comparado ao contralateral, bem como redução significativa do pico de torque de extensores do joelho a $180^{\circ}$ /s, quando comparados ao grupo sem essa patologia. Verificamos que há redução de força muscular de extensores e flexores de joelho em indivíduos com osteoartrose do joelho em fase pré-operatória de artroplastia total.
\end{abstract}

Descritores: Avaliação isocinética, Artroplastia de joelho, Osteoartrose.

\begin{abstract}
The knee osteoarthrosis difficult the routine activities and the deambulation, due to the joint degeneration and the changes in muscle performance that the inferior limb affected presents. The aim of this study was to analyze the muscle concentric peak torque of the extensors and flexors muscle of the knee, through the computadorized dynamometry. It was compared 13 individuals with knee osteoarthrosis due to cartilage degeneration, in preoperative period of total arthroplasty in one of the knees, with 13 individuals without the disease. The results show that individuals affected by the disease presented significant decrease of peak torque of flexors and extensors of the knee indicated for total arthroplasty when compared with the contralateral limb, and also, presented significant decrease in peak torque of knee extensors at $180 \%$ s when compared with the group without the disease. So, it was verified a decrease in muscle strength of extensors and flexors of the knee in subjects with knee osteoarthrosis in preoperative period of total arthroplasty.
\end{abstract}

Key words: Isokinetic evaluation, Knee arthroplasty, Osteoarthrosis. 


\section{Introdução}

A articulação do joelho fornece alta funcionalidade ao indivíduo, tanto em suas atividades rotineiras como caminhar quanto nos exercícios mais complexos encontrados no esporte. Todavia, durante o processo de envelhecimento, esse segmento está suscetível a alterações morfológicas, sobretudo na cartilagem articular, podendo ocasionar uma patologia degenerativa crônica, conhecida como osteoartrose (OA).

Entre as manifestações da OA, observase importante quadro álgico, rigidez articular, redução da amplitude de movimento e déficits de força muscular, prejudicando assim as atividades de vida diária (AVD) dos indivíduos¹. Em sua fase mais avançada, na maioria das vezes há a indicação de uma artroplastia total, a qual foi desenvolvida para reduzir a perda de funcionalidade encontrada em pacientes com doenças degenerativas articulares ${ }^{2}$.

A dinamometria isocinética é usualmente um dos métodos mais acurados para avaliação muscular ${ }^{2}$. Diversas são as maneiras de mensurar e avaliar a atividade muscular; contudo, o método mais exato para este fim é a avaliação isocinética. As mensurações são realizadas por intermédio de um dinamômetro com um sistema computadorizado que permita arcos de movimento em uma velocidade angular constante determinada previamente, o qual oferece informações sobre a dinâmica e o desempenho mecânico dos grupos musculares ${ }^{3}$. O pico de torque é uma das variáveis fornecidas e de fundamental importância para verificar a força e a potência muscular, sendo um excelente indicador do nível máximo de força do indivíduo, não se levando em consideração a $\mathrm{ADM}^{4}$.

Sabe-se que há uma possível redução da força muscular de extensores e flexores em pacientes acometidos pela $\mathrm{OA}^{2,5,6}$. Esse déficit pode ser mensurado por diferentes métodos; no entanto, na literatura revisada não foram encontrados resultados utilizando-se o dinamômetro computadorizado Biodex ${ }^{\mathrm{TM}}$ Multi Joint System 3 Pro.
Para tanto, objetivou-se neste estudo, analisar retrospectivamente o pico de torque muscular isocinético concêntrico dos grupos extensor e flexor do joelho em indivíduos sadios e em outros em fase pré-operatória de artroplastia total, verificando assim qual grupo muscular é de fato o mais acometido pela patologia e também obtendo valores bases de uma possível perda.

\section{Metodologia}

Por meio dos prontuários do Laboratório de Biomecânica da Faculdade de Fisioterapia da Universidade de Passo Fundo, foram selecionados 13 indivíduos - todos encaminhados por dois serviços ortopédicos -, sendo 12 do sexo feminino, e um, do masculino, com diagnóstico de OA por degeneração da cartilagem, com indicação de artroplastia total em um dos joelhos, sem ser revisão de prótese, para compor um dos grupos, denominado grupo 1. Estes sujeitos apresentavam entre 44 e 82 anos, com idade média de 63,61 anos $( \pm 11)$, média de altura de $1,62 \mathrm{~m}( \pm 0,06)$, média de peso de 75,85 kg $( \pm 13,37)$. Pelo cálculo do índice de massa corporal proposto pela Organização Mundial da Saúde (OMS) foi verificado que todos os componentes desse grupo mostravam sobrepeso; oito deles apresentavam seu joelho dominante como o envolvido pela doença; nove eram sedentários e quatro realizavam alguma atividade física (duas a três vezes na semana).

Incluíram-se na análise, as mensurações encontradas em prontuários de mais 13 indivíduos, 12 do sexo feminino, e um, do masculino, para compor o grupo 2, os quais não apresentavam sintomas clínicos compatíveis com OA de qualquer natureza e todos faziam parte de um centro de atividades para a terceira idade. Os sujeitos desse grupo apresentavam entre 58 e 76 anos, com média de idade de 67,85 anos $( \pm 5,85)$, média de altura de $1,56 \mathrm{~m}( \pm 0,08)$, média de peso de $68,6 \mathrm{~kg}( \pm 14,5)$; pelo cálculo do índice de massa corporal citado anteriormente foi verificado que 11 sujeitos apresentam sobrepeso, e apenas dois eram sedentários. 
Foram excluídos os indivíduos que não completaram o teste em decorrência de dor.

Este estudo esteve de acordo com as diretrizes da resolução 196/96 do Conselho Nacional da Saúde e foi aprovado pelo Comitê de Ética da Universidade de Passo Fundo (RS).

Todas as avaliações do pico de torque muscular isocinético ocorreram no dinamômetro computadorizado da marca Biodex ${ }^{\mathrm{TM}}$ modelo Multi Joint System 3 Pro, no Laboratório de Biomecânica da Faculdade de Educação e Fisioterapia da Universidade de Passo Fundo (RS), entre agosto de 2005 e janeiro de 2006. Antes do teste os participantes foram submetidos a um alongamento passivo de MMII (abdutores, adutores, extensores, flexores e tríceps sural), em seguida passavam por aquecimento em bicicleta ergométrica sem carga, durante 5 minutos. Todos os indivíduos foram posicionados na cadeira com encosto inclinado a $85^{\circ}$, estabilizado por cintos no tronco superior, cruzando as espinhas ilíacas anterossuperiores, em volta de uma das coxas e em do tornozelo do membro a ser testado, $2 \mathrm{~cm}$ acima do maléolo medial. O eixo de rotação do dinamômetro era alinhado com o da articulação do joelho, conforme o descrito no manual de aplicações e operações: Biodex ${ }^{\mathrm{TM}}$ Multi Joint System $3 \mathrm{Pro}^{7}$. Realizaram-se as avaliações em duas velocidades angulares, $180^{\circ}$ s e $300^{\circ}$ s. Cada indivíduo executou cinco movimentos completos em cada uma das velocidades, partindo da posição de flexão máxima. Entre cada uma das séries, foi permitido um descanso de 10 segundos. Em seguida, o mesmo procedimento foi realizado no membro contralateral.

Analisaram-se os dados por meio dos recursos de estatística descritiva, e para a comparação entre os grupos, usou-se o teste " $t$ " Student em amostras independentes. Para determinar a significância estatística, utilizou-se $\mathrm{p} \leq 0.05$.

\section{Resultados}

Foram avaliados ao todo 28 prontuários, sendo 13 de indivíduos com diagnóstico de OA por degeneração da cartilagem, com indicação de artroplastia total em um dos joelhos e 13 sem a doença. Os arquivos de dois sujeitos foram excluídos. Eles não conseguiram finalizar o teste por sentirem dor martirizante na articulação e por apresentarem limitação na escala de movimento.

Na mensuração intragrupo do grupo 1, foram verificadas diferenças significativas entre as médias, considerando $\mathrm{p} \leq 0,05$ em praticamente todos os movimentos com exceção da flexão a $180 \%$ s entre o joelho envolvido e o não envolvido. Observaram-se diferenças significativas, desses membros, entre as médias na força do grupo muscular extensor na velocidade de $180^{\circ} / \mathrm{s}$ e $300 \%$ ( $p=0,05$ e $p=0,01$, respectivamente). Além disso, também se verificou diferença significativa entre as médias dos dois membros no grupo muscular flexor, na velocidade de $300 \%$ $(p=0,03)$ (Tabela 1). Sendo assim, os dados sugerem que há diminuição do pico de torque tanto de extensores quanto de flexores em pacientes acometidos por OA, em fase pré-operatória de artroplastia total.

No grupo 2, comparando o joelho envolvido com o não envolvido, observou-se que não houve diferença significativa entre as médias de pico de torque para flexores e extensores a $180^{\circ}$ s, bem como para flexores a $300 \%$ s; entretanto, houve diferença significativa entre as médias nos músculos extensores a 300\% $(\mathrm{p}=0,05)$ (Tabela 2).

Ao comparar o grupo 1 com o 2, não houve diferença significativa entre as médias em nenhum movimento e em nenhuma das velocidades angulares, exceto entre as médias de força do grupo muscular extensor, dos joelhos envolvidos, na velocidade angular de $180 \% \mathrm{~s}(\mathrm{p}=0,04)$ (Tabela 3$)$.

\section{Discussão}

De acordo com Dvir ${ }^{8}$, é contraindicado o uso de velocidades muito baixas em distúrbios patelofemorais ou ligamentares e, considerando que os indivíduos portadores de OA do joelho têm dor e cisalhamento na articulação, usar baixas velocidades nesses indivíduos dificultaria a 
Tabela 1: Diferença entre as médias do pico de torque mensurado no grupo 1

\begin{tabular}{|c|c|c|c|c|}
\hline $\begin{array}{l}\text { Velocidade } \\
\text { angular }\end{array}$ & Movimento & $\begin{array}{c}J^{*}(\mathrm{Nm}) \\
\text { Média }\left(\mathrm{DP}^{* * *}\right)\end{array}$ & $\begin{array}{c}\text { JNE }^{* *}(\mathrm{Nm}) \\
\text { Média }\left(\mathrm{DP}^{* * *}\right)\end{array}$ & $\mathbf{P}$ \\
\hline $180 \% \mathrm{~s}$ & Flexão & $20,01( \pm 11,64)$ & $21,74( \pm 8,84)$ & 0,28 \\
\hline $180 \%$ s & Extensão & $34,48( \pm 16,91)$ & $39,90( \pm 18,19)$ & 0,05 \\
\hline $300 \% \mathrm{~s}$ & Flexão & $22,34( \pm 10,64)$ & $27,33( \pm 9,01)$ & 0,03 \\
\hline $300 \% \mathrm{~s}$ & Extensão & $32,40( \pm 12,17)$ & $37,89( \pm 12,79)$ & 0,01 \\
\hline
\end{tabular}

Tabela 2: Diferença entre as médias do pico de torque mensurado no grupo 2

\begin{tabular}{|c|c|c|c|c|}
\hline $\begin{array}{c}\text { Velocidade } \\
\text { angular }\end{array}$ & Movimento & $\begin{array}{c}\text { JE}^{*}(\mathrm{Nm}) \\
\text { Média }\left(\mathrm{DP}{ }^{* * *}\right)\end{array}$ & $\begin{array}{c}\text { JNE }^{* *}(\mathrm{Nm}) \\
\text { Média }\left(\mathrm{DP}^{* * *}\right)\end{array}$ & $\mathbf{P}$ \\
\hline $180 \%$ s & Flexão & $23,16( \pm 5,50)$ & $23,83( \pm 5,95)$ & 0,36 \\
\hline $180 \% \mathrm{~s}$ & Extensão & $46,12( \pm 16,81)$ & $47,56( \pm 19,01)$ & 0,27 \\
\hline $300 \% \mathrm{~s}$ & Flexão & $25,47( \pm 6,78)$ & $26,90( \pm 7,37)$ & 0,17 \\
\hline $300 \% \mathrm{~s}$ & Extensão & $40,02( \pm 12,86)$ & $36,47( \pm 14,89)$ & 0,05 \\
\hline
\end{tabular}

Tabela 3: Diferença entre as médias do pico de torque entre o grupo 1 e 2

\begin{tabular}{|c|c|c|c|c|c|}
\hline $\begin{array}{l}\text { Velocidade } \\
\text { angular }\end{array}$ & Joelho & Movimento & $\begin{array}{c}\text { Grupo } 1 \\
\text { Média (DP }{ }^{* * *} \text { ) }\end{array}$ & $\begin{array}{c}\text { Grupo } 2 \\
\text { Média (DP }{ }^{* * *} \text { ) }\end{array}$ & $\mathbf{p}$ \\
\hline $180 \% \mathrm{~s}$ & $\mathrm{JE}^{*}$ & Flexão & $20,01( \pm 11,64)$ & $23,16( \pm 5,50)$ & 0,19 \\
\hline $180 \% \mathrm{~s}$ & JE & Extensão & $34,48( \pm 16,91)$ & $46,12( \pm 16,81)$ & 0,04 \\
\hline $180 \% \mathrm{~s}$ & $\mathrm{JNE}^{* *}$ & Flexão & $21,74( \pm 8,84)$ & $23,83( \pm 5,95)$ & 0,24 \\
\hline $180 \% \mathrm{~s}$ & JNE & Extensão & $39,90( \pm 18,19)$ & $47,56( \pm 19,01)$ & 0,15 \\
\hline $300 \% \mathrm{~s}$ & JE & Flexão & $22,34( \pm 10,64)$ & $25,47( \pm 6,78)$ & 0,19 \\
\hline $300 \% \mathrm{~s}$ & JE & Extensão & $32,40( \pm 12,17)$ & $40,02( \pm 12,86)$ & 0,06 \\
\hline $300 \% \mathrm{~s}$ & JNE & Flexão & $27,33( \pm 9,01)$ & $26,90( \pm 7,37)$ & 0,44 \\
\hline $300 \% \mathrm{~s}$ & JNE & Extensão & $37,89( \pm 12,79)$ & $36,47( \pm 14,89)$ & 0,39 \\
\hline
\end{tabular}

realização dos testes, em razão da dor, fraqueza muscular ou limitações na escala de movimento. Berman et al. ${ }^{9}$ realizaram um estudo isocinético no pré e pós-operatório de artroplastia total do joelho, utilizando a velocidade de $60 \%$, considerada uma velocidade angular baixa, havendo uma perda amostral de 17 sujeitos, de um total de 85 , no período pré-operatório, em decorrência dos participantes não conseguirem realizar o teste por dor, fraqueza ou limitações na escala de movimento. Sendo assim, o protocolo estabelecido no Laboratório de Biomecânica, utilizava as velocidades de $180^{\circ} / \mathrm{s}$ e $300 \%$ s para tais indivíduos, as quais são consideradas média e alta velocidade angular, respectivamente.
Stevens et $a .^{10}$ apontaram uma redução da força muscular do quadríceps acometido por OA, quando comparado o lado não acometido, 10 dias antes da realização de artroplastia total de joelho; e Tan et al. ${ }^{5}$ evidenciaram redução do pico de torque isocinético de extensores e flexores do joelho em pacientes com OA, quando comparou com um grupo sem a doença. Esses achados condizem com o presente estudo, em que se verificou uma diminuição do pico de torque em extensores e flexores do joelho na fase pré-operatória de artroplastia total. Identificaram-se também, por meio das anotações de seus exames físicos, sinais clínicos de uma atrofia de quadríceps, as quais condizem com o déficit muscular verificado no 
joelho envolvido dos pacientes desse grupo, em ambas as velocidades angulares adotadas, quando comparado com o membro contralateral.

Rossi et al. ${ }^{6}$ avaliaram a força muscular em 13 sujeitos com OA em um membro, comparando com o contralateral, e verificaram que os extensores e flexores de joelho apresentaram $76 \%$ e $82 \%$ de pico de torque, respectivamente, quando considerado o membro não envolvido. Relacionando esse dado com o presente estudo, foram encontrados resultados similares e comparadas as porcentagens de força muscular nos dois grupos musculares entre os membros dos sujeitos do grupo 1 , observou-se que os extensores e flexores apresentaram $86,41 \%$ e $92,04 \%$ de pico de torque, na velocidade de $180 \%$ s e mostrando $85,51 \%$ e $81,5 \%$, respectivamente, em $300 \%$ s.

Na comparação do pico de torque entre os dois grupos, mesmo sendo o 2 constituído de mais idosos que o 1, talvez não tenha havido diferença significativa na maioria das mensurações de pico de torque em razão deste grupo apresentar somente dois indivíduos com peso normal e dois sedentários, estes últimos diferindo dos outros 11 plenamente ativos. Em decorrência dessa heterogeneidade intragrupos, não foram evidenciadas diferenças significativas entre os dois estudados. Se o grupo 2 fosse, na sua totalidade, plenamente ativo e sem sobrepeso, e o 1 com idades mais próximas, poderia haver uma maior homogeneidade intragrupos e uma maior heterogeneidade intergrupos, dessa forma, talvez fossem encontradas maiores diferenças significativas nas comparações.

Optou-se por utilizar o pico de torque em nossas comparações, pois de acordo com Aquino et al. ${ }^{11}$ a comparação do pico de torque do lado contralateral é fidedigna, visto que ele não encontrou diferença significativa na produção de força, quando comparado o lado dominante e não dominante em mulheres idosas.

O sobrepeso pode ser um fator importante no desenvolvimento de OA. Lewek et al. ${ }^{12}$ encontraram diminuição de força muscular do quadríceps relacionada ao índice de massa corporal em indivíduos acometidos por OA quando submetidos à contração isométrica máxima. Em nosso estudo, não relacionamos a perda de força de quadríceps com o sobrepeso; porém, evidenciamos essa perda por meio da análise do pico de torque e verificamos que todos os sujeitos acometidos pela doença apresentavam sobrepeso. Não obstante, Pozzi e Konkewicz ${ }^{13}$ referem que o excesso de peso provoca um aumento de pressão sobre a articulação do joelho, uma vez que indivíduos obesos apresentam massa muscular menos desenvolvida em virtude de sua condição física.

\section{Conclusão}

Por meio deste estudo, pode-se verificar que existe redução de força muscular de extensores e flexores de joelho em sujeitos acometidos pela OA do joelho em fase pré-operatória de artroplastia total. Entretanto, verificou-se um maior acometimento do grupo muscular extensor, visto que foram encontradas diferenças significativas na comparação entre os membros dos indivíduos com a doença, em ambas as velocidades testadas, indicando assim maior déficit dessa musculatura.

\section{Referências}

1 Thomson A, Skinner A, Piercy J. Artropatias Generativas. In: Fisioterapia de Tidy. $12^{a}$ ed. São Paulo: Santos; 2002. p. 106-27.

2 Aquino MA, Leme LE. Isokinetic dynamometry in elderly women undergoing total knee arthroplasty: a comparative study. Clinics. 2006;61:215-22.

3 Siqueira CM, Pelegrini FR, Fontana MF, Greve GM. Isokinetic dynamometry of knee flexors and extensors: comparative study among non-athletes, jumper athletes and runner athletes. Rev Hosp Clin São Paulo. 2002;57:19-24.

4 Brown LE, Weir JP. Recomendação de procedimentos da Sociedade Americana de Fisiologia do Exercício (ASEP) I: avaliação precisa da força e potência muscular. Revista Brasileira de Ciência e Movimento. 2003;11:95-110. 
5 Tan J, Balci N, Sepici V, Gener FA. Isokinetic and isometric strength in osteoarthrosis of the knee - a comparative study with healthy women. Am J Phy Med Rehabil. 1995;14:364-9.

6 Rossi MD, Brown LE, Whitehurst M. Knee extensor and flexor torque characteristics before and after unilateral total knee arthroplasty. Am J Phy Med Rehabil. 2006;85:737-46.

$7 \quad$ Biodex $^{\mathrm{TM}}$ Multi Joint System 3 Pro. Manual of applications/operations. New York; 2002.

8 Dvir Z. Isocinética - avaliações musculares, interpretações e aplicações clínicas. Barueri: Manole; 2002.

9 Berman AT, Bosacco SJ, Israelite C. Evaluation of total knee arthroplasty using isokinetic testing. Clin Orth Rel Res. 1991;271:106-13.
10 Stevens JE, Mizner RL, Snyder-Mackler L.

Quadriceps strength and volitional activation before and after total knee arthroplasty for osteoarthritis. J Orth Res. 2003;21:775-9.

11 Aquino MA, Leme LE, Amatuzzi MM, Greve JM, Terreni AS, Andrusaitis FR, Nardelli JC. Isokinetic assessment of knee flexor/extensor muscular strength in elderly women. Rev Hosp Clin Fac Med São Paulo. 2002;57:131-4.

12 Lewek MD, Rudolph KS, Snyder-Mackler L. Quadriceps femoris muscle weakness and activation failure in patients with symptomatic knee osteoarthritis. J Orthop Res. 2004;22:110-5.

13 Pozzi JFA, Konkewicz ER. Joelho do Adulto. In Hebert S, Xavier R. Ortopedia e traumatologia: princípios e prática. $3^{\mathrm{a}} \mathrm{ed}$. Porto Alegre: Artmed; 2003. p. $444-72$. 